\title{
Les fondations politiques mémorielles en France : pour la déiense d'une histoire orientée ?
}

Il y a maintenant bientôt dix ans, dans un véritable pamphlet sur les défis auxquels fait face l'historien, Christophe PROCHASSON livrait son sentiment sur les rapports entre le travail d'historien et les organisations mémorielles en plein

L'auteur : Pierre-Emmanuel Guigo est maître de conférences en histoire à I'Université Paris Est Créteil (UPEC) développement :

\begin{abstract}
Ce que demandent les groupes mémoriels, quels qu'ils soient, aux historiens, est moins le résultat de leurs recherches, par définition toujours ambivalentes, toujours irrécupérables, que les arguments en faveur de leur juste cause'.
\end{abstract}

Depuis, l'enjeu paraît toujours aussi grand, voire même encore plus présent. Historien du politique, Christophe PROCHASSON avait peutêtre en tête les fondations politiques mémorielles qui commençaient à exister. Par fondation politique mémorielle, nous entendons donc les organismes structurés qui ont pour but de défendre la mémoire d'un groupe ou d'un acteur politique individuel impliqués dans la vie politique française passée. Ces organismes font désormais partie du quotidien de l'historien du politique. Face à une diminution des financements pour la recherche et pour la recherche en histoire politique plus spécifiquement, face à la raréfaction des postes universitaires dans ce domaine, à la diminution des étudiants suivant ce type de cours, les fondations - nous utilisons le terme de fondation par commodité de langage, elles ne sont pas toutes des fondations au sens juridique du terme - politiques mémorielles deviennent une bouée vitale pour une histoire politique en plein déclin². Apparues il y a environ quarante ans, leur essor participe donc de la montée des revendications mémorielles bien mise en valeur par Pierre NORA en 1984 dans son introduction aux Lieux de mémoire ${ }^{3}$. L'historien, dont le travail est d'écrire le « récit du passé, [qui] instaure d'entrée de jeu une distance ${ }^{4}$ navigue ainsi

\footnotetext{
1 Prochasson Christophe, L'empire des émotions: les historiens dans la mêlée, Paris, Demopolis, 2008 , p. 210. 2 LE BEGUEC Gilles, "L'histoire politique à l'heure des défis : controverse et perspectives », in SIRINELLI JeanFrançois et allii. Les historiens français en mouvement, Paris, PUF, 2015, pp. 151-161.

3 NORA Pierre, «Introduction » in NORA Pierre (dir.), Les lieux de mémoire, tome 1, Paris, Gallimard, 1984. 4 Joutard Philippe, Histoire et mémoire: conflits et alliances, Paris, La Découverte, 2013, p. 15.
} 
dans des eaux où sa parole est contestée par ces " entrepreneurs de mémoire ${ }^{5}$ " et il peut être amené à se justifier, voire connaître des retombées judiciaires dans les cas les plus extrêmes ${ }^{6}$. La mémoire, parcellaire par essence, relève de l'affect et non de l'analyse froide qui doit être celle de l'historien?. C'est sans doute avec l'émergence de "lois mémorielles » que l'opposition entre historiens et mémoires a été la plus vive. Plusieurs collectifs d'historiens se sont mis en place pour protester de ce qui apparaissait comme une immixtion de l'État dans la recherche historique. Ce que l'on observe de manière croissante dans d'autres domaines de l'histoire paraît encore plus valable en ce qui concerne l'histoire politique. Ce ne sont plus seulement des mouvements, aussi actifs soient-ils auquel l'historien fait face, mais des organismes bénéficiant par leur objet de relations privilégiées avec le pouvoir politique, ce qui rend les risques d'obstruction beaucoup plus probables.

La prolifération de ces fondations rend-elle le travail de l'historien du politique plus difficile? Quelle marge de manœuvre peut-on trouver pour continuer à écrire l'histoire du politique sans pression?

Nous avons choisi de nous concentrer sur dix de ces fondations:

- Fondation DE GAULLE ;

- Fondation Jean JAURÈs,

- Institut Pierre Mendès France,

- Association MichelRocard.org,

- Institut François MitTERRAND,

- Fondation Napoléon,

- L'Office Universitaire de Recherche socialiste (OURS),

- Fondation Gabriel PÉRI,

- Institut Georges POMPIDOU,

- Institut Tribune socialiste.

Au cours de notre recherche, nous avons pu néanmoins découvrir d'autres de ces organisations, souvent plus confidentielles, mais il ne nous a pas été possible de les étudier plus en profondeur.

5 Buscatto Marie, "Voyage du côté des "perdants " et des "entrepreneurs de mémoire » ", Ethnologie française, 2006/4 (Vol. 36), pp. 745-748.

6 On peut notamment penser à la plainte à l'encontre d'Olivier PÉTRÉ-GRENOUILLÉ suite à la publication de son livre Les traites négrières, essai d'histoire globale, Paris, Gallimard, 2004. 
Pour mener à bien cette recherche, nous avons bénéficié d'une position d'observation privilégiée au sein de trois de ces fondations. Tout d'abord, nous avons eu l'occasion de travailler - participation à des séminaires, colloques, écriture d'articles, et même parfois travaux rémunérés - pour la Fondation Jean JAURÈs et pour l'Institut François MitTerRand dès 2011. Depuis 2014, nous sommes également le principal responsable des activités de l'association MichelRocard.org, en raison de notre thèse portant sur cette figure du socialisme français dans la deuxième moitié du xxème siècle. Cette insertion nous a permis de voir de près le fonctionnement de ces organisations et tout particulièrement les relations qu'elles peuvent entretenir avec les historiens. Nous avons pu avoir accès à des sources exclusives comme la comptabilité ou encore les agendas de ces fondations.

Par nos responsabilités au sein de l'association MichelRocard. org et nos recherches, nous avons aussi eu l'occasion de travailler avec l'Institut Tribune socialiste et l'Office universitaire de recherche socialiste (OURS). Nous complétons cette enquête de terrain avec une étude des sites internet de ces fondations, ainsi qu'une analyse de leurs publications. Enfin, nous avons entrepris l'élaboration de deux questionnaires, l'un à destination d'historiens que nous avons côtoyé au cours de nos recherches, pour la plupart spécialistes de l'histoire politique. Sur cinquante questionnaires envoyés, nous avons reçu 31 réponses. L'autre questionnaire s'adresse à des responsables de ces fondations mémorielles identifiés par nos contacts ou les organigrammes en ligne. Sur l'ensemble des fondations nous avons reçu seulement quatre réponses (OURS, ITS, Fondation Napoléon, Institut François MITTERRAND).

\section{Ies fondations mémorielles politiques en voie d'expansion}

Les revendications mémorielles et ce qu'elles font au travail de I'historien est un sujet déjà exploré depuis plusieurs décennies. De la colonisation aux génocides, en passant par l'esclavage, les historiens voient leur parole discutée, contestée. Dans le domaine politique, le sujet a moins fait l'attention. II faut bien dire que l'histoire politique en général ne suscite plus la déférence qu'elle eut par le passé.

La précarité dans laquelle elle est entrée la rend d'autant plus vulnérable aux pressions mémorielles, nous y reviendrons. Dans ce mouvement d'expansion des revendications mémorielles, la vie politique a pris toute sa place. Pour les acteurs ou les organisations politiques, il est devenu capital de valoriser leur trace. Non pas que leurs prédécesseurs n'aient pas cherché à le faire également. Les véhicules 
utilisés étaient différents. La désignation d'héritiers, la fondation de dynasties, l'empreinte architecturale, la rédaction de mémoires, étaient des outils plus communs.

Peu actives jusqu'aux années 1980, on ne compte plus depuis les fondations dédiées à la valorisation d'un groupe ou d'un homme politique. Chaque ancien président ou ancien Premier ministre entend désormais défendre lui-même sa trace ou par le biais de son entourage, alors que le champ libre ou presque était laissé jusque-là aux historiens. La toute première apparaît en 1952 avec la Société des amis de Léon BLUM, dont l'activisme reste toutefois très modéré et faiblement impérialiste pour l'historien. La figure de Guy MOLLET va être décisive à gauche pour l'émergence de ce type de structure. En 1969 il crée l'Office universitaire de recherche socialiste (OURS) qui doit permettre la conservation des archives de la SFIO et leur valorisation. Par la suite, en 1975, à sa mort, est créée une fondation qui porte son nom, suivie ensuite par l'association des amis de Victor FAY, ancien militant communiste puis du PSU. C'est surtout à partir des années 1980, et plus particulièrement la fin de cette décennie, que ces fondations se développent vraiment. La loi sur le mécénat de 1987, va favoriser l'expansion de fondations d'un nouveau style, bénéficiant de bien plus de ressources. II ne s'agit plus cette fois de petites associations aux travaux confidentiels, mais de fondations reconnues d'utilité publique et vivant largement des subventions de l'État et dotées de moyens de communication sans précédent - une revue, congrès, colloques, etc.). La première à bénéficier de ce statut est la Fondation Napoléon créée à l'initiative de l'industriel Martial LAPEYRE, qui a fait fortune dans le commerce de bricolage, en 1987. Suivent en 1989 l'Institut Georges POMPIDOU et la Fondation DE GAULLE. À gauche, on trouve d'abord la Fondation Jean JAURĖS créée par Pierre MAUROY qui, au-delà de la valorisation de l'œuvre de JAURÈs et de son créateur, sert de centre de réflexion pour la gauche, ainsi que quelques années plus tard (1996) l'Institut François MITTERRAND, créé à la mort de l'ancien président. Dans les années 2000, c'est l'Institut Tribune socialiste, rassemblant les anciens du Parti socialiste unifié - l'Institut rassemble également une constellation de petites associations du même type comme l'institut Edouard DePREUX ou l'Association des amis de Victor et Paule FAY - qui est créé, ainsi que peu après l'Association Michel Rocard.org (2014).

Comme le montre cette énumération, ces fondations sont plus nombreuses à gauche, même si celles qui peuvent être classées à droite de l'échiquier politique (DE GAULLE, Napoléon, POMPIDOU) sont en général beaucoup mieux dotées que la multitude de petites associations financées par cotisations et dons que l'on trouve à gauche (exception faites de l'Institut François MITTERRAND et de la Fondation Jean JAURÈs). Cela rejoint l'analyse de Perrine CanaVAggio qui réflé- 
chissant aux archives des partis montrait un plus fort tropisme des partis de gauche que des partis de droite pour leurs archives et leur histoire ${ }^{8}$. Elle soulignait que l'un des moyens pour conserver ces archives était justement la création d'instituts comme l'Office universitaire de recherche socialiste. On peut également remarquer qu'à droite c'est toujours autour d'une personnalité politique (DE GAULLE, POMPIDOU, Napoléon) que se crée l'association, alors qu'à gauche, et ce conformément au refus plus grand de la personnalisation qui la caractérise, les collectifs sont plus souvent honorés (OURS, ITS, Fondation Jean JAURÈS) 9 .

Le cadre législatif et ses évolutions ont aussi sans doute favorisé l'essor de ces fondations mémorielles. La législation française est en effet plutôt souple sur la création d'associations. Selon la loi du ier juillet 1901, il suffit d'une simple déclaration en préfecture indiquant un titre, un objet, un siège et la liste de ses administrateurs pour créer une association. Le seul coût est la publication de sa création au Journal Officiel pour 44 euros. Ce statut n'a jamais cessé de voir son succès s'accroître au cours du xxème siècle. Depuis le début des années 2000 , on compte même jusqu'à 70.000 nouvelles créations d'associations par $\mathrm{an}^{10}$. Ces organisations peuvent également être reconnues "d'intérêt général », ce qui leur permet d'émettre des reçus fiscaux pour leurs donateurs. Pour l'obtenir, elles doivent faire une demande auprès de l'administration fiscale qui l'évaluera en fonction du caractère philanthropique, scientifique, social, humanitaire, éducatif ou culturel, et uniquement si l'activité de l'association est désintéressée et non lucrative. Grâce à ce statut, les donateurs vont en effet pouvoir retirer $66 \%$ de leurs dons de leur impôt sur le revenu et même de l'impôt sur la fortune (article 200 du Code général des impôts). Les réseaux politiques étant souvent constitués de personnes concernées par ces dispositifs, les fondations vont donc devenir un bon moyen de défiscalisation. Certaines obtiennent même d'être reconnues d'utilité publique par une décision du Conseil d'État. Beaucoup plus difficile à obtenir - il s'agit d'un décret du ministère de l'Intérieur après décision du Conseil d'État suite à la constitution d'un dossier beaucoup plus exigeant -, ce statut permet à l'association de bénéficier en plus du rescrit fiscal de la possibilité de recevoir des donations et des legs.

\footnotetext{
8 Canavaggio Perrine. "Une enquête de l'Association des archivistes français ", La Gazette des archives, n¹48, 1990. Les archives des partis politiques, pp. 11-22.

9 Berstein Serge et Rudelle Odile, Le modèle républicain, Paris, PUF, 1992, 432 p. 
Comme nous l'avons vu, la date de 1987 est décisive dans l'émergence de fondations et en particulier des plus importantes. En effet, c'est en 1987 que le statut de fondation est strictement défini par la loi, grâce à la loi sur le mécénat, avec des avantages bien plus importants qu'une simple association de loi 1901. Selon l'article 18 de la loi du 23 juillet 1987, « La fondation est l'acte par lequel une ou plusieurs personnes physiques ou morales décident l'affectation irrévocable de biens, droits ou ressources à la réalisation d'une œuvre d'intérêt général et à but non lucratif. "Ce nouveau statut est particulièrement attractif pour les organisations naissantes. II permet en effet, outre le rescrit fiscal, les dons et les legs, de bénéficier de subventions publiques de l'État et d'acquérir des biens immobiliers, y compris sans lien avec les activités de la fondation. La durée de la fondation est illimitée ce qui assure une pérennité à toute épreuve à l'organisme. Pour obtenir la reconnaissance comme fondation d'utilité publique, il faut toutefois constituer un capital de départ (750.000 euros au minimum), avoir des statuts très précis et un programme pour les trois prochaines années. Cette appellation, particulièrement attractive, est donc réservée aux plus grands organismes qui se sont eux aussi multipliés ces vingt dernières années, la Fondation Charles DE GAULLE, la Fondation Jean JaURĖs, la Fondation Napoléon, l'Institut François MITTERRAND en témoignent.

Ces différents avantages, en particulier fiscaux, permettent à ces fondations d'avoir des budgets conséquents et dont le monde de la recherche bénéficie d'une partie, voire de la quasi-totalité. Face aux baisses de crédit que connaît le monde universitaire, cette nouvelle ressource peut se révéler très attractive pour les historiens. La Fondation Jean JAURÈS a ainsi un budget annuel de 2.730 .000 euros dont $18 \%$ est consacré au secteur " histoire et mémoire », soit 487.000 euros" $^{11}$ La Fondation Napoléon qui n'a pas le rôle de think tank de la Fondation Jean JAURÈS, voit la quasi-intégralité de son budget annuel dédié à la valorisation mémorielle, avec un budget annuel de plusieurs millions d'euros ${ }^{12}$. Le budget de la Fondation DE GAULLE est lui aussi supérieur à un million d'euros (1,7 million) par an ${ }^{13}$. Seul l'Institut François MITTERRAND a un budget inférieur à un million d'euros, puisque le budget moyen annuel de cette fondation d'utilité publique

\footnotetext{
11 "La Fondation », Fondation Jean JAURÈS, disponible à l'adresse suivante : https://jean-jaures.org (consultée le 29 septembre 2017).

12 "Comptes annuels de l'exercice 2016 soumis à l'approbation du conseil d'administration du 20 juin 2017 ", juin 2017, disponible à l'adresse suivante : https://fondationnapoleon.org (consultée le 29 septembre 2017). 13 Rapport d'activité 2016, Fondation Charles DE GAULLE, 2016, disponible à l'adresse suivante: http://www. charles-de-gaulle.org (consultée le 29 septembre 2017).
} 
avoisine les 300000 euros $^{14}$. Les autres organismes n'ayant pas le statut de fondation ont des ressources nettement moins importantes. Le budget annuel de l'Association MichelRocard.org est d'environ 40.000 euros par $a^{15}$, et il doit être sans doute inférieur pour les autres associations.

Enfin, si les fondations politiques mémorielles se sont largement développées ces dernières années en France, il s'agit aussi d'une tendance plus internationale. Les fondations françaises se sont semble$t$-il inspirées des fondations étrangères, dont certaines beaucoup plus anciennes. En Allemagne, ces fondations sont en effet très nombreuses et beaucoup mieux dotées qu'en France. Presque tous les hommes d'État d'envergure nationale ont leur stiftung. Leur généalogie remonte au début du XXème siècle, la fondation Friedrich EBERT (du nom du premier président de l'Allemagne entre 1919 et 1925) a été créée en 1925. Les principales sont même soutenues par le Bundestag comme les fondations Otto vON BISMARCK, Friedrich EBERT, Theodor Heuss, Konrad AdenAUER et Willy BRANDT. Leurs moyens n'ont rien de commun avec les fondations françaises, puisqu'elles s'appuient largement, en plus des dons, sur les subsides publics dans le cadre d'une vraie politique de valorisation de la mémoire du pays (budget du délégué du Gouvernement fédéral à la Culture et aux Médias) ${ }^{16}$. Il est nécessaire de préciser qu'elles n'ont pas qu'un rôle mémoriel, mais qu'elles servent aussi de think tank pour le parti politique dont elles sont proches. Mais leur rôle mémoriel concernant la personnalité qui porte leur nom est non négligeable. Elles gèrent souvent des lieux mémoriels, organisent des expositions, des parcours éducatifs, éditent leurs ouvrages, remettent des prix, financent des recherches, et gèrent des centres d'archives, tout en salariant des dizaines de personnes ${ }^{17}$. À l'exception des fondations Napoléon et DE GAULLE, rares sont celles à pouvoir en faire de même en France. À titre de comparaison, le budget moyen par an des fondations Friedrich EBERT et Konrad ADENAUER avoisine 100 millions d'euros, là où il n'est que de 3 millions pour la Fondation Jean JAURÈs, l'une des plus importantes françaises ${ }^{18}$. Cet écart est aussi dû à leur rôle diplomatique et de représentation de la culture allemande dans le monde ${ }^{19}$. En Grande-Bretagne, aussi,

\footnotetext{
14 Entretien avec Georges SAUNIER, responsable de l'Institut François MITTERRAND, 16 octobre 2017. 15 Documents comptables pour le Conseil d'administration de l'Association MichelRocard.Org. 16 "Ce qui nous relie ", Découvrir l'histoire à travers des biographies, disponible à l'adresse suivante : $\underline{\text { http:// }}$ www.politikergedenkstiftungen.de (consultée le 29 septembre 2017).

17 Synthèse $n^{\circ} 81$, Fondation Robert SCHUMAN, disponible à l'adresse suivante: https://www.robert-schuman. eu (consultée le 29 septembre 2017). 18 lbid. 
on constate un développement de ces fondations avec notamment la Margareth THATCHER foundation, du nom de l'ancienne Premier ministre, créée en 1991, la Lloyd GEORGE Society (Premier ministre entre 1916 et 1922) et le Churchill Archives Center, créé en 1973 et adossé à une fondation internationale. Aux États-Unis, il n'y a pas de fondations mémorielles au sens strict, mais des think tanks jouent ce rôle. Evelyne JosLaIN parle ainsi de Vanity tanks «créés en l'honneur d'une personnalité politique ou bien fondés sur un legs politique qui n'a pas pu être mené à terme ${ }^{20}$ ". Parmi les plus célèbres on compte le FRANKLIN Institute, la HOOVER Institution ou le NIXON Center, qui à côté de leurs activités de recherche valorisent l'héritage de ces personnalités marquantes de la vie politique américaine ${ }^{21}$. La HOOVER Institution on War, Revolution and peace possède ainsi de nombreux fonds d'archives privés ${ }^{22}$. Ces modèles étrangers sont très présents dans l'esprit des dirigeants des fondations françaises. L'Institut François MITTERRAND revendique par exemple sur son site un travail commun avec la Fondation KREISKY, du nom de l'ancien chancelier social-démocrate autrichien (1970-1983), Bruno KREISKY. On voit donc que si les fondations politiques mémorielles sont importantes, elles le sont toutefois moins que dans d'autres pays. Ce qui avait amené à un rapport, puis à une proposition de loi du sénateur Jacques OUDIN en 20042005 afin de renforcer le statut des fondations françaises ${ }^{23}$. Certes moins puissantes que leurs homologues étrangers, ces fondations n'en restent pas moins des acteurs incontournables de l'histoire politique contemporaine.

\section{Une tentation pour les historiens}

De plus en plus présentes dans le paysage politique et historique ces fondations rencontrent rapidement le parcours du chercheur en histoire politique. Comme nous avons pu le voir, la plupart de ces organisations dédient tout ou partie de leur activité à la recherche sur la période dont elles entretiennent la mémoire. Elles participent de l'émergence de ce que l'on appelle désormais la Public History, c'està-dire l'évocation, divulgation de l'histoire dans l'espace public et ce

\footnotetext{
20 JosLain Evelyne, L'Amérique des think tanks, Paris, L'Harmattan, 2006, p. 84.

21 Ibid.

22 lbid.

23 OUdin Jacques, Les Fondations démocratiques à vocation politique en France : rapport au Premier ministre, La documentation française, décembre 1997, 281 p., disponible à l'adresse suivante : http://www. ladocumentationfrancaise.fr (consultée le 29 septembre 2017).
} 
en relation avec le monde universitaire ${ }^{24}$. La plupart décernent une bourse aux chercheurs, pour des thèses et même plus souvent pour des mémoires de recherche. L'Institut François MITTERRAND décerne ainsi depuis dix ans un prix du meilleur mémoire portant sur l'histoire politique de la période ${ }^{25}$. Doté de 1.000 euros, le prix permet aussi au jeune chercheur de publier son travail, via les partenariats de l'Institut avec le monde de l'édition. La Fondation Jean JAURĖs s'est aussi dotée d'un prix pour les mémoires de recherche sur l'histoire du socialisme et du monde ouvrier avec un montant de 1.000 euros aussi, s'accompagnant également d'une publication du travail ${ }^{26}$. Mais c'est sans doute la Fondation Napoléon qui offre les prix les plus attractifs, avec pas moins de trois prix par année dotés de 5.000 euros $^{27}$. Ces prix sont en général décernés suite à la décision d'un jury composé d'historiens spécialistes de la période (Alain BERGoUnIOUX pour la Fondation Jean JAURĖs, Frédéric Bozo pour l'Institut MITTERRAND, Eric ANCEAU pour la Fondation Napoléon), et d'anciens collaborateurs ou de proches de la personnalité ou du groupe politique en question. $22 \%$ de nos 30 sondés ont reçu un prix d'une de ces fondations. Certaines de ces fondations, c'est le cas en particulier de la Fondation Napoléon, soutiennent même des jeunes chercheurs pour l'élaboration de leur thèse via des bourses d'étude. Chaque année, la Fondation décerne ainsi six bourses pour des jeunes doctorants de 7500 euros chacune ${ }^{28}$. Ainsi, notre questionnaire permet de voir que $15 \%$ de nos enquêtés ont déjà bénéficié d'une de ces bourses.

Ces dotations deviennent particulièrement attractives dans un monde de la recherche où les crédits se raréfient et où les bourses de recherche se réduisent. Outre ces financements accordés aux recherches sélectionnées, les fondations sollicitent aussi des chercheurs pour des travaux rémunérés. Ainsi, parmi nos trente questionnés, $25 \%$ ont exercé une activité rémunérée pour l'une ou plusieurs de ces fondations. II s'agit le plus souvent d'écriture de textes dans le cadre de la divulgation des ressources de la fondation - la création d'un site internet comme "François MitTerRAND, le verbe et l'image »,

\footnotetext{
24 KELLEY Robert, «Public History : its origins, nature and prospects », The Public Historian, 1978, vol. 1, pp. 16-28.

25 "Le prix Master de l'Institut François MitTeRRAND », Institut François MitTerand, disponible à l'adresse suivante : http://www.mitterrand.org (consultée le 29 septembre 2017).

26 "Le prix de la Fondation Jean JAURÈs », Fondation Jean JAURÈs, disponible à l'adresse suivante : https:// jean-jaures.org (consultée le 29 septembre 2017).

27 "Les Prix d'histoire », Fondation Napoléon, disponible à l'adresse suivante : https://fondationnapoleon. org (consultée le 29 septembre 2017). 
de l'Institut François MitTerRand, qui permet de valoriser les interventions télévisées de l'ancien président de la République, à chaque fois commentées par un historien spécialiste du sujet ${ }^{29}$ - ou de la vulgarisation scientifique. Certaines de ces fondations emploient également des stagiaires venus du monde de la recherche. Autant d'emplois qui suppléent à la raréfaction du nombre de postes stables d'enseignants-chercheurs, notamment depuis l'autonomie des universités en $2008^{30}$.

Structure de financement pour les chercheurs et en particulier les jeunes chercheurs, ces fondations offrent également un soutien à la valorisation scientifique. Elles sont souvent dotées de revues «scientifiques" - Recherche socialiste pour l'OURS, La Lettre de l'Institut pour l'Institut François MITTERRAND, Napoleonica pour la Fondation Napoléon, Espoir pour la Fondation DE GAULLE - pour lesquelles les chercheurs sont sollicités et qui publient beaucoup plus facilement que les revues à comité scientifique liées à des organismes de recherche universitaire. En ce qui concerne notre questionnaire, sur 30 répondants, $40 \%$ avaient déjà écrit pour une de ces revues. Sans même y écrire, ces revues recensent les ouvrages publiés sur la question, ce qui permet d'accroître la notoriété des publications. L'OURS présente une vingtaine d'ouvrages par mois, ainsi que les thèses soutenues sur l'histoire du mouvement ouvrier ou du socialisme ${ }^{31}$. Elles aident également les chercheurs à publier leurs travaux par des bourses de publication ou encore via les prix précédemment cités. Ainsi, selon notre questionnaire, 30\% des répondants ont déjà reçu une aide à la publication, utile dans un monde de l'édition, lui aussi touché par la crise.

Outre les publications, ces fondations organisent des événements visant à présenter les travaux scientifiques sur la période, et notamment de nombreux colloques. L'Institut François MITTERRAND a organisé pas moins de six colloques pour le centième anniversaire de la naissance de François MITTERRAND en 2016. La Fondation Jean JAURÈS organise ou co-organise elle aussi plusieurs colloques à travers toute la France par an. L'Association MichelRocard.org co-organise avec l'Institut Tribune socialiste un colloque sur Michel RocARD et le PSU, le 24 novembre 2017, ainsi que pour mai 2017, un colloque sur les années

\footnotetext{
29 «MITTERAND », Ina.fr, disponible à l'adresse suivante $:$ http://fresques.ina.fr/mitterrand/ (consultée le 29 septembre 2017).

30 Une chute de 33\% du nombre de postes ouverts au concours selon une étude statistique faite par Guillaume MIQUELARD-GARNIER, disponible à l'adresse suivante : http://blog.educpros.fr (consultée le 29 septembre 2017). Des enquêtes plus récentes donnent même une baisse de $38 \%$ sur six ans, si l'on inclut les chiffres de 2017.
} 
Rocard à Matignon (1988-1991) avec l'Institut d'Etudes Politiques de Paris. La Fondation DE GAULLE, quant à elle, organise des séminaires autour d'un thème chaque année, alors que l'Institut Georges POMPIDOU est à l'origine de journées d'études de conférences. Ces ressources sont donc particulièrement désirables dans un monde de la recherche qui voit sans cesse ses crédits baisser. Cela avait même donné lieu à un "Cri d'alarme » de l'Académie des Sciences en $2013^{32}$. Sur les dix dernières années, le budget stagne, alors même qu'il progresse dans la plupart des pays développés et en voie de développement. Classée au dixième rang mondial en 2002, la France n'est plus que 16ème en ce qui concerne la recherche ${ }^{33}$. Le budget 2017 prévoit ainsi 337 millions d'euros en moins pour l'Enseignement supérieur et la recherche. Même le CNRS, navire-amiral de la recherche française est touché par la baisse des crédits et la raréfaction des postes. À l'Université également, les postes d'enseignants-chercheurs se font plus rares ${ }^{34}$. Plus de la moitié de notre réseau de chercheurs avait déjà participé à une activité scientifique de ces fondations. Grâce aux moyens qui sont les leurs, ces fondations parviennent également à publier des actes de colloque, ce qui s'avère pourtant de plus en plus dur en l'absence de financement préalable. $80 \%$ de nos enquêtés ayant participé à un colloque de ces fondations ont ensuite vu leur communication publiée. Le chercheur en nouvelles technologies Pierre MOUNIER considérait ainsi en 2008 que «la publication d'actes de colloque en sciences humaines et sociales sur support papier est une activité condamnée, en partie parce qu'elle est commercialement suicidaire ${ }^{35}$."

Enfin, comme nous avons pu le voir précédemment, ces organisations sont aussi souvent des centres d'archives. Pour l'historien du politique, elles sont incontournables pour accéder aux sources qui distinguent le travail d'historien. $70 \%$ de nos enquêtés ont déjà fait des demandes d'accès aux archives de ces fondations. L'OURS conserve plus d'une centaine de fonds de militants et d'organisations - Parti socialiste SFIO 1905-1969, Fédération de la gauche démocrate et socialiste 1965-1969. La Fondation Jean JAURĖS qui travaille en

\footnotetext{
32 " 'Cri d'alarme' de l'Académie des sciences sur le financement de la recherche ", Institut de France, Académie des sciences, 2013, disponible à l'adresse suivante: http://www.letudiant.fr (consultée le 29 septembre 2017).

33 RenARD Camille, «Financement de la recherche: la France, élève médiocre », France culture, 24 mai 2016 , disponible à l'adresse suivante : https://www.franceculture.fr (consultée le 29 septembre 2017).

34 HUET Sylvestre, "Budget de la recherche 2018 : la vérité est dans le bleu ", \{Sciences\}, 8 octobre 2017, disponible à l'adresse suivante : http://huet.blog.lemonde.fr (consultée le 17 avril 2018). 
étroite relation avec l'OURS (les deux fondations sont dans les mêmes locaux) conserve elle les archives socialistes depuis 1969, ainsi que les archives du groupe socialiste, les archives du Mouvement des jeunes socialistes, et de plusieurs anciens dirigeants du PS : Pierre MAUROY (1949-2013), François MITTERRAND (1971-1981), Lionel JoSPIN (1981-1988), Claude ESTIER (1970-1986), Pierre BÉRÉGOVOY (1973-1979), notamment ${ }^{36}$. L'Institut Tribune socialiste conserve les publications du PSU (Tribune socialiste, Critique socialiste), les affiches du même parti, ainsi que des fonds d'anciens militants comme Marc HEURGON ${ }^{37}$.

La Fondation Napoléon et l'Association MichelRocard.org, ainsi que la Fondation Jean JAURĖs pour une partie de ses fonds, ont fait le choix du numérique. Pour les deux premières, l'intégralité de leurs fonds a été numérisée et mise en ligne sur un site prévu à cet effet et offrant une interface de recherche. Pour la Fondation Napoléon, il s'agit de la correspondance de Vivant Denon, des travaux du Conseil d'Etat durant le Premier Empire (3666 documents), la correspondance de Napoléon avec Bigot DE PRÉAMENEu (ministre des Cultes), l'album Houdetot (des portraits par un jeune auditeur du Conseil d'État), des documents sur la proclamation de l'Empire, issus de la bibliothèque de la Fondation ${ }^{38}$. Pour l'association MichelRocard.org, c'est une partie des archives de Michel RoCARD, extraite de son fonds aux Archives Nationales (680AP), des cartons de la Fondations Jean JAURÈs, et d'archives conservées à son domicile qui a été numérisée et mise en ligne sur le site homonyme de l'association. On y trouve l'intégralité des discours de Michel RocARD depuis 1980 jusqu'en 2011, la revue Convaincre, ainsi que de nombreuses photographies. Enfin, la Fondation Jean JAURĖS a développé un imposant site de recherche sur lequel on peut retrouver tous les comptes rendus de congrès depuis 1939, de conseils nationaux du PS, les périodiques édités par le parti (L'Unité, Vendredi, Le Poing et la rose entre autres), ainsi qu'un millier d'affiches.

La Fondation DE GAULLE conserve, pour sa part, près d'un millier de cartons, la plupart étant liés au RPF, le premier parti du général DE GAULLE et à ses militants. Elle conserve aussi des manuscrits de discours, des correspondances, des collections de journaux ainsi que presque tous les dossiers des voyages du général DE GAULLE en

\footnotetext{
36 "Responsables socialistes ", Archives socialistes, disponible à l'adresse suivante : http://www.archivessocialistes.fr (consultée le 29 septembre 2017.

37 "Le centre de documentation ", Institut tribune socialiste, disponible à l'adresse suivante : http://www. institut-tribune-socialiste.fr (consultée le 4 octobre 2017).

38 "Informations », Napoleonica.org, disponible à l'adresse suivante : http://www.napoleonica.org (consultée le 30 septembre 2017
} 
province et à l'étranger de 1944 à 1970. La correspondance adressée à la famille DE GAULLE à son décès se trouve également dans leur centre d'archives rue de Solférino ${ }^{39}$. L'Institut François MITTERRAND conserve également des archives de la période précédant la victoire à l'élection présidentielle de 1981, ainsi que de sa présidence, mais sans qu'un inventaire détaillé soit accessible sur internet. L'Institut Georges POMPIDOU conserve lui les témoignages de proches de Georges POMPIDOU, à travers un programme d'archives orales (180 entretiens avec les collaborateurs de Georges POMPIDOU), les écrits et discours de Georges POMPIDOU, une bibliothèque thématique centrée sur les années 1960-1970, la presse d'époque (notamment Le Monde diplomatique $)^{40}$. À l'image des fondations Jean JAURÈs, Napoléon et Rocard, l'Institut Georges POMPIDOU s'est également doté d'un site accueillant les discours du président de la République, numérisés. La Fondation Gabriel Péri conserve elle les bibliothèques de militants ou de chercheurs sur le communisme ${ }^{41}$. Toutes ces fondations possèdent également une salle de lecture ou de consultation, indispensable pour en faire un lieu de recherche.

Enfin, la vie de chercheur étant aussi faite de sociabilité, ces fondations aident les chercheurs, et en particulier les plus jeunes, à se constituer un réseau de connaissance. Sur 22 répondants, 18 considèrent que la participation aux activités de ces fondations a contribué à améliorer leur réseau de recherche. Certains soulignent que ce fut pour eux l'occasion de rencontrer des collègues spécialistes du même sujet. Même si cela ne ressort pas de notre enquête, ces fondations sont également un moyen pour les chercheurs de côtoyer des témoins qu'ils pourront ensuite interroger pour leurs recherches, voire leur apporter des archives. Concernant notre cas personnel, c'est le travail pour l'association MichelRocard.org qui nous a permis de mieux approcher l'ancien Premier ministre et d'accéder à ses archives personnelles conservées à son domicile ${ }^{42}$.

S'appuyant sur des ressources financières et matérielles importantes, ces fondations politiques mémorielles sont donc devenues pour les historiens du politique des partenaires presque incontournables. Dans le cadre de leurs travaux, ils doivent souvent passer

39 «La bibliothèque de la Fondation », Fondation Charles DE GAULLE, disponible à l'adresse suivante : http:// www.charles-de-gaulle.org (consultée le 29 septembre 2017).

40 "Accueil du public », Institut Georges POMPIDOu, disponible à l'adresse suivante : https://www. georges-pompidou.org (consultée le 2 octobre 2017).

41 " Archives \& Histoire du communisme et du mouvement ouvrier », Fondation Gabriel PÉRI, disponible à l'adresse suivante : http://www.gabrielperi.fr (consultée le 2 octobre 2017). 
par elles pour financer leurs recherches, les documenter, les valoriser. Elles se situent ainsi à tous les niveaux de l'activité de recherche. Les historiens que nous avons interrogés sont très majoritairement enthousiasmés par le travail avec ces fondations. 27 répondent positivement en ce qui concerne leur travail d'historien du politique et à la question de savoir s'ils sont favorables à la création d'autres fondations, 60\% répondent positivement. Sur 26 répondants, un seul ne souhaite pas prolonger ses recherches avec ces fondations.

\section{l'historien muselé ?}

Derrière la bonne image générale de ces fondations politiques mémorielles qui ressort de notre questionnaire auprès des historiens, notre $21^{\mathrm{e}}$ question permet de voir les craintes qui existent néanmoins à l'égard de l'emprise de ces organisations. Nos historiens sont bien conscients que leur but n'est pas uniquement de servir la recherche scientifique de manière désintéressée. La plupart relève que le principal risque est l'orientation de la plume du chercheur par la fondation en question. Leur vocation étant avant tout mémorielle, elles cherchent à valoriser l'œuvre d'un homme politique ou d'une organisation. Ainsi, un des questionnés relève que : "ces fondations mènent un véritable combat mémoriel pour faire triompher la mémoire de leurs grands hommes (justifier leurs actions principalement). Elles possèdent les financements, orientent les recherches, permettent de se constituer des réseaux pour les chercheurs mais elles semblent dangereuses pour certains aspects de l'histoire souvent tumultueuse de leurs 'grands' hommes (il n'y a d'ailleurs que des hommes blancs dans ces fondations). Elles font le 'best of' des meilleurs moments d'untel et jettent le reste dans la grande poubelle de l'oubli ». Le risque est donc en étant trop dépendant de ces fondations de faire une histoire partielle, voire hagiographique, conformément à une mémoire " oublieuse "43 des moments sombres. À l'occasion d'un travail collectif pour la valorisation du fond d'une de ces fondations, lors d'une réunion collective, un chercheur avait ainsi fait la remarque qu'un point manquait sans doute concernant la personnalité en question, c'était une rubrique " affaires » dans la production finale. Celui-ci développait l'argumentaire qu'il ne s'agissait pas de faire une rubrique à scandale, mais plutôt de présenter l'état de la recherche sur un domaine qui a fait couler beaucoup d'encre concernant cette personne. Mais l'entourage de l'acteur politique en question n'a pas souhaité donner de suite à cette suggestion du chercheur. 
Un des enquêtés fait d'ailleurs remarquer qu'il a décidé de cesser toute collaboration avec certaines fondations suite à des pressions. Mais, cela semble néanmoins marginal si l'on en croit nos répondants. La plupart assurent même avoir bénéficié d'une totale liberté de travail et dans leurs conclusions, y compris lorsqu'ils ont bénéficié d'aides pour leur publication. Pour améliorer encore les rapports entre ces fondations et les historiens, il est donc nécessaire qu'elles soient plus transparentes dans leur mode de fonctionnement. Presqu'aucun des enquêtés n'est au courant des modes de financement de ces fondations. Un des répondants souligne même qu'une indépendance du "secteur historique/archivistique " permettrait d'éviter toute forme d'entrave à la liberté de l'historien. Un autre conseille de faire entrer plus de chercheurs dans la direction de ces fondations, au détriment des anciens collaborateurs et des proches. C'est un mouvement que les fondations semblent d'ailleurs adopter ${ }^{44}$. Elles écartent de plus en plus l'entourage pour le cantonner à des fonctions honorifiques, privilégiant la constitution de comités scientifiques composés majoritairement d'universitaires ${ }^{45}$. $30 \%$ de nos répondants participent d'ailleurs au conseil scientifique d'une de ces fondations.

L'autre risque le plus souvent évoqué par nos enquêtés tient aux sources détenues par ces fondations. Elles peuvent choisir de n'ouvrir qu'une partie des fonds et selon des critères qui ne relèvent pas de la loi, contrairement aux archives publiques. Ce risque est encore accru par le statut flou de ces archives. Plusieurs de nos enquêtés relèvent avoir vu des documents qui devraient plutôt être conservés aux Archives Nationales. En effet, depuis la loi du 3 janvier 1979, les archives produites dans le cadre étatique doivent être déposées obligatoirement dans les centres d'archives publiques. Toutefois, rares sont les répondants à faire part de difficultés à consulter les archives détenues par ces fondations. Ils pointent toutefois un manque de clarté sur les fonds conservés qui peut être amélioré, notamment par la mise en ligne d'inventaires détaillés, ce que la Fondation Napoléon et l'Institut Georges POMPIDOU ont entrepris récemment. Un de nos enquêtés souligne également que la multiplication des fondations peut amener un éparpillement des sources rendant ainsi la recherche plus complexe. Mais au contraire, l'un des répondants souligne une accessibilité plus facile dans le centre d'archives d'une de ces fondations qu'aux archives nationales... La revue Vingtième siècle faisait 
d'ailleurs remarquer que ces fondations permettaient aussi de sauver des fonds d'archives voués à disparaître en raison de la saturation des archives publiques ${ }^{46}$.

Certains historiens de notre enquête soulèvent aussi une question plus profonde qui tient cette fois à la direction donnée à la recherche scientifique. Selon l'extrait d'un commentaire cité plus haut, ces fondations contribuent à perpétuer la mémoire d'une vie politique dominée par une catégorie sociale, sexuelle et raciale, écartant du même coup tous les «subalternes " pour reprendre l'appellation que leur donne un courant de recherche ${ }^{47}$. L'historien du politique de demain, comme l'homme ivre cherchant ses clés, se focaliserait sous le lampadaire sécurisant des financements et archives assurés, oubliant ainsi tous les «dominés». C'est pourtant contre ce type d'histoire des puissants que l'école des Annales, en grande partie fondatrice de la recherche contemporaine en histoire s'était élevée au début du XXème siècle. Le risque est grand de rester dans une histoire archaïque et paresseuse comme Henri HAUSER, en 1904, la dénonçait déjà :

\footnotetext{
Toutes nos histoires antérieures à 1789, et même un certain nombre d'histoire publiées au XIXe siècle, ne méritent que très imparfaitement le nom d' 'histoires de France'. En réalité ce sont, cousues bout à bout les biographies de nos rois [...]. L'histoire ne pouvait devenir démocratique sans devenir économique, car les classes qu'elle amenait sur le devant de la scène étaient des classes de travailleurs et de producteurs. [...] [Le peuple] n'avait-il pas été aussi, d'aventure, l'auteur de l'histoire de l'ancienne France ? ${ }^{48}$
}

En regardant cette fois le détail des répondants à notre questionnaire, en lien avec leur profil, on constate que ce sont surtout les chercheurs les plus âgés et occupant les positions les plus prestigieuses dans le monde universitaire qui font preuve de précaution à l'égard de ces fondations. Les jeunes chercheurs, ceux qui bénéficient donc en général des bourses, prix de recherche et aides à la publication, sont au contraire les plus enthousiastes, ce qui peut inquiéter pour l'indépendance des chercheurs à venir. 


\section{Conclusion}

Les fondations politiques mémorielles sont donc devenues des acteurs importants de la recherche scientifique en histoire politique. Un cadre légal plutôt favorable, des ressources financières importantes leur ont permis de soutenir une recherche en histoire politique en voie de rétractation. Elles interviennent à tous les niveaux de l'activité de recherche, de la contribution financière, à la vulgarisation scientifique, en passant par la collecte de sources pour l'historien, l'organisation de colloques, et la publication de travaux via leurs revues ou des ouvrages. À travers notre enquête auprès d'un échantillon d'historiens que nous avons sollicité, nous pouvons voir qu'elles apparaissent avant tout comme une aubaine pour eux, en particulier pour les plus jeunes chercheurs en quête de financements et de publications. Contrairement à l'opposition stricte entre histoire et mémoire telle que développée par Pierre NoRA et nombre des auteurs qui l'ont suivi, il faut plutôt voir la situation sous l'angle de la collaboration. L'idée d'une histoire rationnelle, dépassionnée et d'une mémoire irraisonnable et orientée tient plutôt de l'idéal-type que de la réalité contemporaine de la recherche. Nicolas OfFENSTADT souligne ainsi que « cette distinction n'est pas sans poser problème tant l'historien est lui-même inséré dans les enjeux de mémoire, qu'il soit partie prenante de leur définition ou bien pris à témoin par les porteurs des identités en jeu $»^{49}$.

Il faut donc plutôt penser les rapports entre historiens et mémoires - puisque le pluriel s'impose ici - sous l'angle de la coopération. Philippe JouTARD souligne ainsi les complémentarités entre histoire et mémoires. Reprenant les propos d'Eric VIGNE il montre comment souvent dans la recherche "la mémoire instruit l'histoire " ${ }^{50}$. Les fondations mémorielles ne joueraient-elles pas un rôle pionnier dans la conservation et l'étude des matériaux d'une histoire présente toujours sujette à la suspicion de beaucoup d'historiens? C'est peut-être un nouveau rapport au public qu'il faut forger comme le prône d'ailleurs l'histoire publique, en plein développement, comme en témoigne la création d'un département qui lui est consacrée à l'Université Paris Est Créteil ${ }^{51}$. 
Ces fondations posent aussi la question de la place de l'historien dans la cité. Peut-il sortir de sa tour d'ivoire pour devenir un " expert " au service d'une cause? C'est une position qui ne semble pas choquer notre échantillon, mais qui a largement été rejetée jusque-là par l'historiographie, ne voyant guère de salut en dehors de la "recherche fondamentale ». Lucien FEBVRE n'était-il pas le premier à s'écrier qu'une histoire qui sert est une histoire "serve ${ }^{52}$ ". Mais cette posture du chercheur éloigné de ton engagement est en bonne partie une vue de l'esprit. L'historien vit dans son temps et se nourrit de ce qui l'entoure. Norbert ELIAS étudiant cette question voyait plutôt un continuum entre la recherche fondamentale et l'engagement qu'un fossé infranchissable ${ }^{53}$.

Les enquêtés perçoivent toutefois les dangers à s'en remettre totalement à ces fondations dont les buts sont clairement différents de ceux de l'historien. Leur laisser trop de place dans l'activité scientifique peut conduire à une histoire orientée, visant essentiellement à valoriser la personne ou le groupe en question et s'appuyant sur les sources qu'elles veulent bien présenter. Ces fondations doivent donc rester des entrepreneurs de mémoire "modestes " selon l'expression de Philippe JOUTARD. Ne pas prétendre représenter la seule vision possible de l'histoire et chercher à s'imposer au travail du chercheur ${ }^{54}$. C'est aussi une mémoire qui doit accepter les remises en cause de la part des historiens pour améliorer encore plus le travail de ces fondations. Perfusion vitale dans une situation de pénurie de la recherche, ces fondations ne doivent donc pas se substituer à des investissements publics, seuls à même de garantir l'objectivité de l'historien. Hubert BONIN faisait des remarques similaires à propos des histoires d'entreprise qui ont souvent vocation à créer une légende dorée des compagnies en instrumentalisant les travaux d'historiens ${ }^{55}$. Pouvoir s'appuyer sur une allocation de recherche, des crédits pour les publications, et des archives conservées et mises à la disposition selon les critères du droit public restent la clé pour éviter la confusion croissante entre histoire et mémoire.

52 FebVre Lucien, Leçon inaugurale à l'université de Strasbourg, 1919.

53 ELIAS Norbert, Engagement et distanciation, Paris, Fayard, 1993, 258 p.

54 JouTARd Philippe, op. cit., p. 274.

55 BonIN Hubert, "L'histoire d'entreprise ne "sert » à rien! ", Communication et organisation, n7, 1995. 